\title{
Use of kinetic flow energy liquids for vibration of local turbulizers in pipe heat exchangers
}

\author{
Rakhimjan Babakhodjaev ${ }^{1}$, Nazim Tashbaev $^{1}$ and Djonreed Mirzaev ${ }^{1}$ \\ ${ }^{1}$ Tashkent State Technical University named after Islam Karimov, Heat power engineering and nuclear power plants Depertment, \\ Universitetskaya 2, Tashkent, Uzbekistan
}

\begin{abstract}
Annotation. This issue of using certain part of the kinetic energy of the flow for imparting vibration for special local turbulators (LT), consisting of an elastic thread with strung on it through a certain distance volume elements (made of a special material) of various geometric configuration installed in the tubes of the heat exchanger with fixing one end of the thread at the inlet, and the other end remains free. The results of numerical and experimental studies are presented, which show the effectiveness of the use of LT for intensifying the processes of hydrodynamics and heat transfer.
\end{abstract}

\section{Introduction}

Analysis of known sources on the topic under study allows us to note many effective methods for intensifying heat transfer, such as: artificial turbulization of the flow in the near-wall zone using various methods (transverse protrusions and grooves, stamping on ribs, pipe finning and threading, twisting flow inside coiled oval pipes using twisted tapes and screw inserts; controlled separation of the boundary layer in the transverse flow of tube bundles and a combination of intensification methods $[1,2,3,4,5,6]$. Intensification of heat transfer in flows fluid due to turbulization is usually accompanied by an increase in the hydraulic resistance coefficient. Obtaining the ratio $\mathrm{Nu} / \mathrm{Nu}_{S S}>\xi / \xi_{S S}$ (thermal power and hydraulic resistance, ss - smooth surface) allows to reduce the volume of the apparatus and its cross-sectional area $[7,8,9]$. Also known are the results of research on hydrodynamics and heat transfer when flowing around a surface with dimples. Such surfaces are characterized by the relative depth and diameter of the hole, as well as the density of the location of the holes and the tightness of the channel. Smoothing the edges of the dimples leads to a decrease in the hydraulic resistance of the channels with dimples $[10,11]$. The vibration of the heat exchange surface intensifies the heat transfer, however, along with this, the heat exchanger itself is subjected to vibration and its reliability necessary to take into account not only the efficiency, but also the manufacturability and contamination of the surface. of operation decreases. When choosing a method for intensifying heat transfer, it is

\section{Physical model and hydrodynamic principles}

LT consists of an elastic wire 1 and hollow elements 2 strung on it at a certain distance (Fig. 1.). The LT is inserted into the heat exchanger tubes with one end attached at the tube inlet. The other end of the wire remains free.

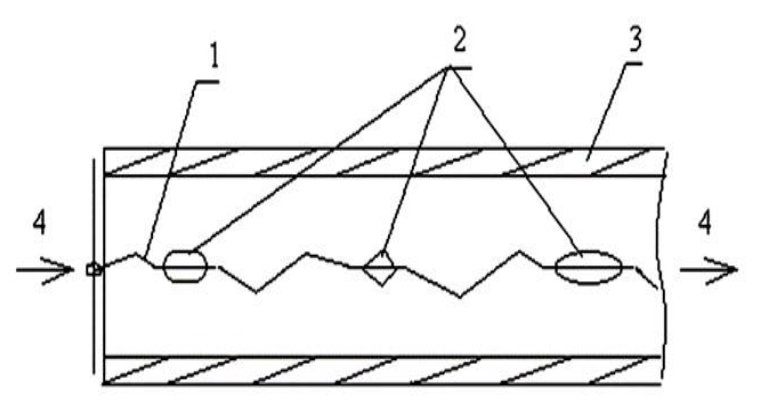

Figure: 1. A tube with local turbulators: 1 - elastic wire; 2 - elements of LT; 3 - tube; 4 - direction of movement of raw water

•Corresponding author: rachimjan@mail.ru 
The coolant flow 4, washing the surface of the elements 2 , pulls the wire back to a certain distance, and the wire elastic force, exceeding the kinetic energy of the fluid flow, returns the hollow elements back to their original position. Then, under the action of the kinetic force of the flow, the wire starts to tighten again and when the elastic force becomes greater than the force of the flow, the wire takes on its original shape. Thus, the wire with empty-body elements makes a forward-reverse motion. In addition, the hollow elements under the action of the flow and due to the geometric shape make a transverse movement inside the tube. The superposition of the forward-backward and transverse motion gives the LT vibration. The vibration frequency of the hollow elements depends on the elasticity of the wire, on the specific gravity of the hollow elements, their external configuration, as well as on the flow rate of the coolant. The creation of flow turbulization with the use of LT consumes some of the kinetic energy of the fluid flow. When using forced turbulization, of particular importance is the hydraulic resistance provided by the LT to the fluid flow, which requires research to determine the rational relationships between the Reynolds and Nusselt criteria and to compare the efficiency of the developed LT design with known modern designs $[12,13,14,15]$.

\section{Computer modeling and numerical analysis}

A numerical study of the turbulization of coolant flows with the help of LT in the tubes of the heat exchanger was carried out, where the parameters of the operating experimental setup were taken as the initial data for the calculation: internal diameter of the tube $-20 \mathrm{~mm}$; tube length 400-1100 mm; coolant speed 0.01-0.15 m/ s. In a computer model, 4 different designs of local turbulators are investigated. Taking into account the accepted parameters of the installation, a computer

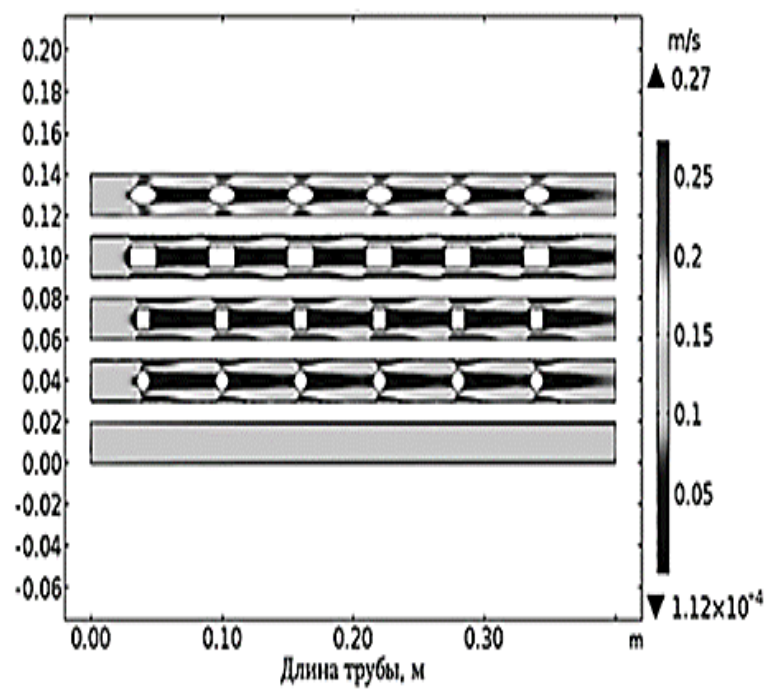

Figure: 2. Distribution of the velocity field, V [m/s] model was compiled in the Comsol Multiphysics software environment with the conditions of an incompressible fluid and wall impermeability. The

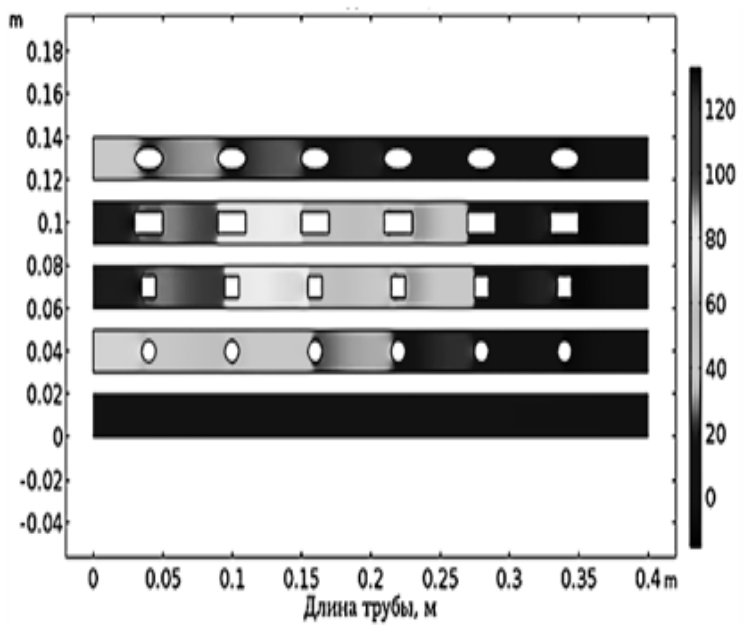

Figure: 3. Distribution of the flow pressure field, $\Delta \mathrm{P}[\mathrm{Pa}]$

model of turbulent heat and mass transfer in a liquid includes the Navier-Stokes equations (the law of conservation of momentum), continuity (the law of conservation of the mass of a liquid), and diffusion transfer of a scalar quantity (the law of conservation of mass).

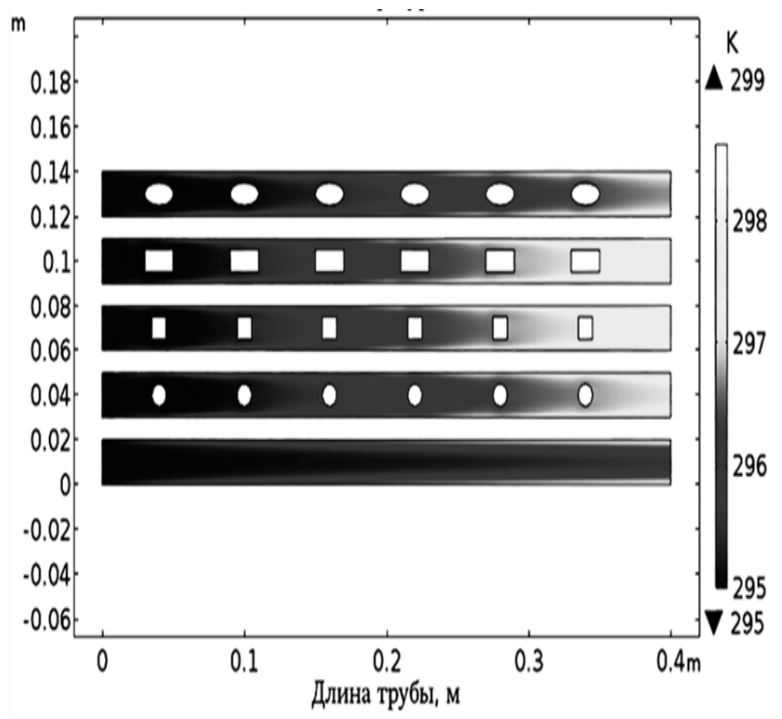

Fig. 4. Temperature field distribution, $\mathrm{T}[\mathrm{K}]$.

As result of the performed numerical experiments, data was obtained characterizing the effect of the RT installed in the tubes on the distribution of the field of velocities, pressures and temperatures in the tubes, presented in Figures 2, 3 and 4. Analysis of the results of numerical experiments showed that some LT designs strongly change the distribution pattern value of speed, head loss and temperature in the tube. This influence is especially evident in the near-night zone. 
Influence of fluid flow turbulization on the processes of hydrodynamics and heat transfer

Figures 5, 6 and 7 show graphical dependencies obtained by the method of a numerical experiment and data from a physical experiment.

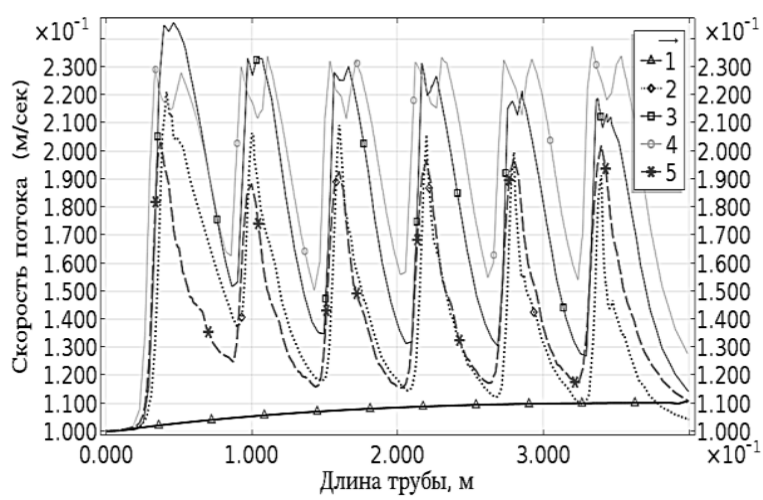

Figure: 5. Graph of fluid velocities in a tube with LT.

From the velocity, graph (Fig. 5) it can be seen that the fluid flow passes into a turbulent state due to the influence of the LT elements. The lower relatively

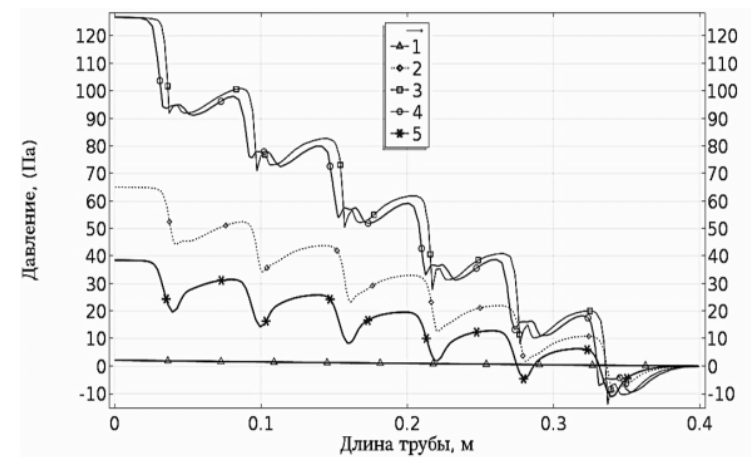

Figure: 6 Graph of fluid pressure loss along the length of the tube with LT.

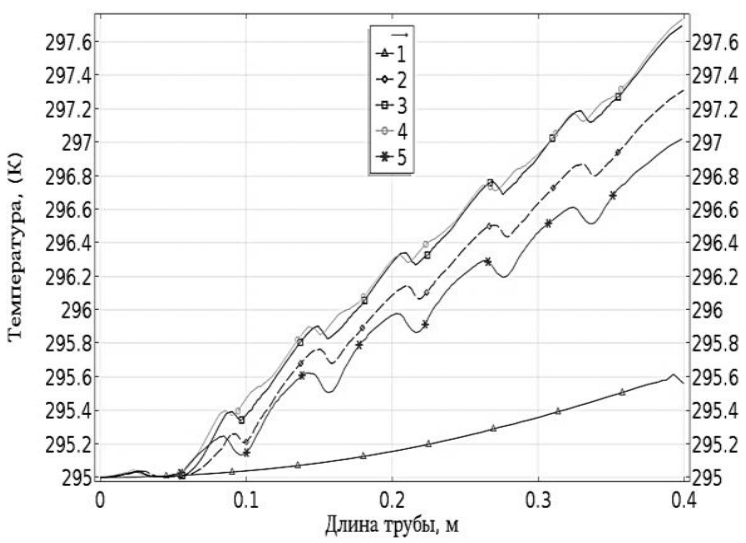

Figure: 7. Field of isotherms of liquid in a tube with LT

straight line was obtained for a tube without LT under the same flow regimes. The speed jumps occur at the location of the LT elements. The shift of the jumps to the left or right, sometimes down or up the graph, is explained by the existence of the vibration of the LT elements. In fig. 6 shows the head loss along the length of the tube. It was determined that each jump on the graph falls on the locations of the LT elements, where the flow is compressed. Curves of changes in the temperature of the liquid along the length of the tube is shown in Fig. 7, which show that the increase in the temperature of the liquid significantly depends on the presence of LT. It should be noted that different designs of LT have different effects on the change in the temperature of the liquid.

\section{Experimental setup}

The author has developed constructions of an effective LT, investigated on experimental installations, the diagram of one of which is shown in Fig. 8. The experiments were carried out according to the following technique. Fluid flow hydrodynamics and the effect of LT structures on the process were investigated on the "cold" model. The liquid flow is passed through a glass tube 1 without LT and the flow regimes are determined. Then, for the selected flow regime, the investigated design of the LT is installed into the tube, and a dye is added to the flow for clarity of the flow pattern. Flow regimes in the "cold" mode are transferred to the "hot" part of the experimental setup. The investigated design of the LT is rearranged into the metal tube 3 and the tube 6 , also made of metal, but free from LT, is switched on. Metal tubes 3 and 6 have $\mathrm{d}=22 \mathrm{~mm}$ and are heated by electric heaters to create a heat flow of a certain power from the outside. The experiments were carried out to determine the effect of LT on the efficiency of heat transfer. The experimental data for various designs of the LT with tube 3 were compared both with each other and with the data obtained for tube 6. The setup is equipped with appropriate measuring and control devices.

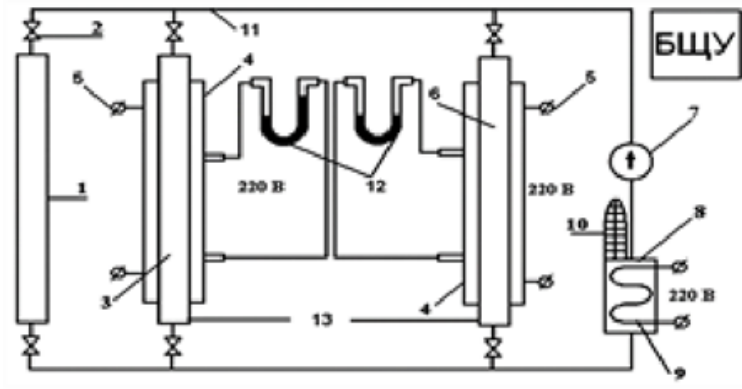

Figure: 8 . Schematic diagram of the experimental setup: 1-glass tube; 2 - valve for regulating water flow; 3- metal tube with LT; 4- thermal insulation with an internal heat source; 5- supply of electrical energy to 4; 6- metal tube without LT; 7- water pump; 8 and 9 - electrically heated thermostat; 10- water flow meter: 11- connecting pipelines; 12- manometers; БЩУ - Control Panel Unit.

\section{Physical experiment results}


Figures 9, 10 and 11 show the results of an experimental study to determine the influence of the developed LT designs on the efficiency of the processes, and also show the calculated equations characterizing the indicated curves.

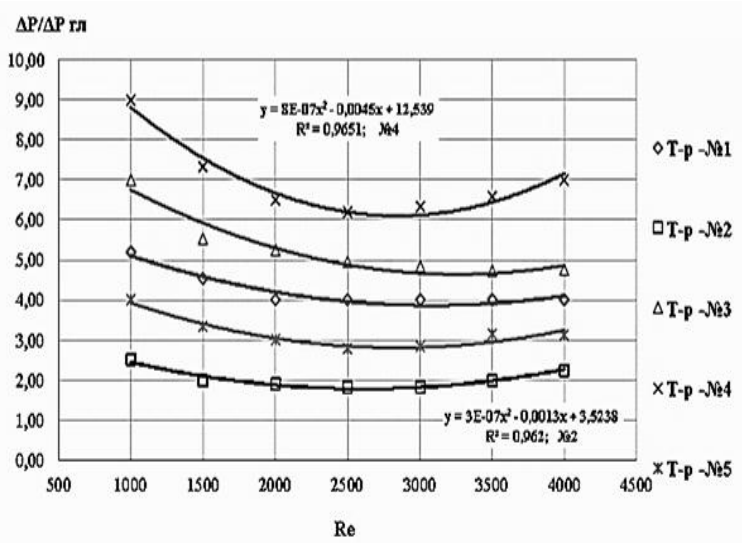

Figure: 9. Curves $\Delta \mathrm{P} / \Delta \mathrm{P}_{\mathrm{SS}}$ depending on $\mathrm{Re}$ for various designs of LT

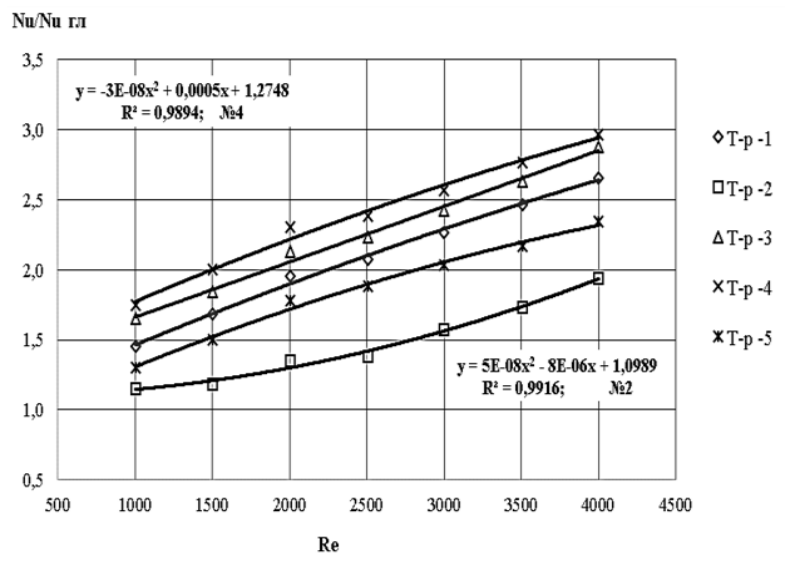

Figure: 10. Curves $\mathrm{Nu} / \mathrm{Nu}_{\mathrm{SS}}$ depending on $\mathrm{Re}$ for various LT designs

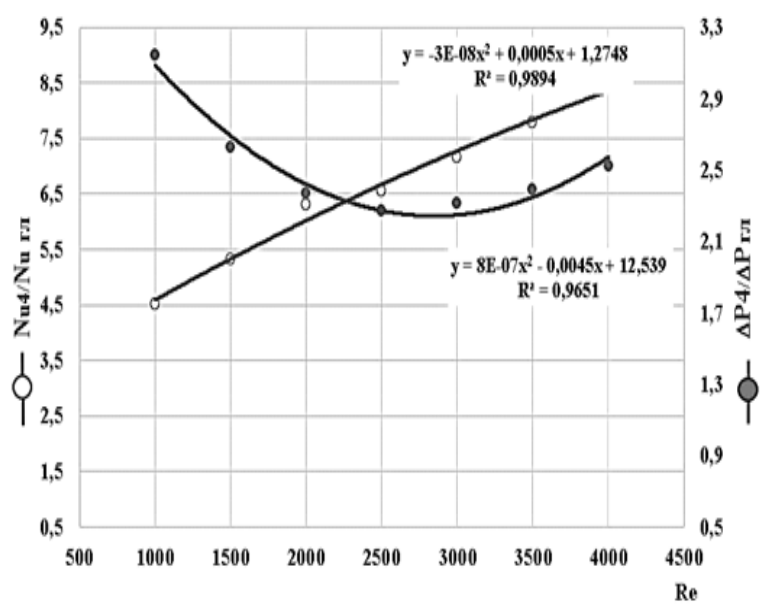

Figure: 11. Graphic determination of rational design parameters of LT
Fig. 9 that with an increase in the Re number, the ratio $\Delta \mathrm{P} / \Delta \mathrm{P}_{\mathrm{SS}}$ decreases monotonically, and in a certain interval of Re values practically does not change, and then begins to increase. This is explained by the fact that the presence of LT at certain values of the flow rate leads first to a decrease and then to an increase in resistance, while maintaining an increase in the efficiency of heat transfer (Fig. 10)

In fig. 11 shows a graphical method for determining the area of rational design and operating parameters of the considered LT, which makes it possible to increase the efficiency of the heat exchange equipment.

\section{Conclusion}

Modeling the processes of intensification of hydrodynamics and heat transfer in tubular heat exchangers confirmed the validity of the choice of the physical model and research methodology $[13,14,15$, 16]. The resulting turbulence model makes it possible to predict the structure of the flow in the tubes, to determine the details of the secondary flows and the movement of the turbulized zones as a result of vibration. The design of the developed LT is technologically advanced and easy to manufacture, accessible and inexpensive for installation and operation. The obtained empirical dependencies allow calculating the values of the heat transfer coefficients with an acceptable degree of error.

\section{Literature}

1. E.K. Kalinin, G.A. Dreitser and S.A. Yarkho Intensification of heat exchange in the channels. M.: Mashinostroenie, (1990). 200 p.

2. E.K. Kalinin, G.A. Dreitser, I.Z. Kopp and other. Effective heat exchange surfaces. M .: Energoatomizdat, (1998). 407 p.

3. Yu.F. Gortyshov, V.V. Olimpiev and I.A. Popov The efficiency of industrially effective heat transfer intensifiers (Review. Analysis. Recommendations). Izvestiya RAS, Energetika, (2002), 3, p. 102-118.

4. G.A. Dreitser, A.S. Myakochin Influence of the geometric shape of turbulators on the efficiency of intensification of convective heat exchange in pipes // Thermal Engineering, (2002). 6. p.57-59.

5. Industrial Heating Principles, Techniques, Materials, Applicants, and Design. Yeshvant V. Deshmukh. Published in 2005 by CRC Press Taylor \& Francis Group 6000 Broken Sound Parkway NW, Suite 300 Boca Raton, FL 33487-2742 .p. 775.

6. Process heat transfer principles, applications and rules of thumb. Robert W. Serth, Thomas G. Lestina. 2014 Elsevier Inc. All rights reserved. Academic Press is an im print of Elsevier. The Boulevard, Lang-ford Lane, Kidlington, Oxford OX5 1GB, UK. p. 616. 
7. V.K. Migai. Improving the efficiency of modern heat exchangers. L .: Energy, (1980), 143 p.

8. Yu.A. Kuzma-Kichta. Heat transfer intensification methods. M.: MEI, (2001), 112 p.

9. G.A. Dreitser, B.V. Dzyubenko and R.I. Yakimenko. Intensification of heat transfer and analysis of methods for comparing the thermohydraulic efficiency of heat transfer surfaces. // Proceedings of the Second Russian National Conference on Heat Transfer. M.: MEI. (1998). 6. p. 99-102.

10. A.A. Khalatov, I.I. Borisov and S.V Shevtsov. Heat and mass transfer and thermohydraulic efficiency of vortex and swirling flows. Kiev: Institute of Technical Thermophysics, NAS of Ukraine. (2005), 500 p.

11. A.S. Vlasenko. Increasing the efficiency of heat exchangers through the intensification of heat transfer on surfaces with spherical recesses // Abstract of a dissertation of a doctor of technical sciences. M.: MPEI, (2011). 36 p.

12. R.P. Babakhodjaev and others. Turbulizing device for heat exchange tubes. IAP 04514. Patent for invention of the Republic of Uzbekistan. Bull. 6, (2012).

13. R.P. Babakhodjaev, A.A. Sadiev, Kh.A. Kuchimov and L.M. Eshkuvatov Laboratory research facility for studying the processes of maximum utilization of the heat of exhaust flue gases. Actual problems and prospects for the development of construction, heat and gas supply and energy supply: Proceedings of the VII full-time International scientific-practical conference / Ed. F.K. Abdrazakova. Saratov: FGBOU VO Saratov GAU, (2018). p. 44-47.

14. R.P. Babakhodjaev Intensification of the processes of hydrodynamics and heat transfer in the tubes of shelland-tube devices with the use of local turbulators. Coal heat power engineering: Problems of rehabilitation and development: Abstracts. Alushta, (2006). p. 51-52.

15. R.P. Babakhodjaev, U.Kh. Ibragimov, D.A Mirzaev and L.M. Eshkuvatov. Increasing the cooling capacity of tubular heat exchangers and reducing the rate of scale and deposits build-up. Collection of articles. III AllRussian Scientific and Practical Conference "Energy and Energy Saving: Theory and Practice". Kuzbass GTU. (2017). p.145-150 p.

16. R.P. Babakhodjaev, A.O. Shakirov and Tashbaev N.T. Study of fluid flow hydrodynamics in the near-wall region of heat exchanger channels in order to reduce the rate of sedimentation of solid impurities. Energy: management, quality and efficiency of energy resources use: Proceedings of the 8th International Scientific and Technical Conference. Blagoveshchensk. (2015). p.361363. 